\title{
Optimum driving condition for lift creating cylinder by plasma actuators based on wind tunnel evaluation-based design
}

\author{
Masahiro Kanazaki* \\ Graduate School of System Design, \\ Tokyo Metropolitan University, \\ Tokyo, Japan \\ Fax: +81-42-585-8606 \\ Email: kana@sd.tmu.ac.jp \\ *Corresponding author

\section{Takashi Matsuno, Kengo Maeda and Hiromitsu Kawazoe} \\ Graduate School of Engineering, \\ Tottori University, \\ Tottori, Japan \\ Fax: +81-857-31-6708 \\ Email: matsuno@mech.tottori-u.ac.jp \\ Email: b081054@gmail.com \\ Email:kawazoe@mech.tottori-u.ac.jp
}

\begin{abstract}
Kriging-based genetic algorithm (GA) was employed to optimise the parameters of the operating conditions of plasma actuators (PAs). In this study, the lift maximisation problem around a circular cylinder was considered. Two PAs were installed on the upper and the lower side of the cylinder. This problem was similar to the airfoil design, because the circular has potential to work as airfoil due to the control of flow circulation by the PAs with four design parameters. The aerodynamic performance was assessed by wind tunnel testing to overcome the disadvantages of time-consuming numerical simulations. The developed optimisation system explores the optimum waveform of parameters for $\mathrm{AC}$ voltage by changing the waveform automatically. Based on these results, optimum designs and global design information were obtained while drastically reducing the number of experiments required compared to a full factorial experiment. An analysis of variance and a scatter plot matrix were introduced for design knowledge discovery. According to the discovered design knowledge, it was found that duty ratios for two PAs are an important parameter to create lift.
\end{abstract}

Keywords: plasma actuator; genetic algorithm; efficient global optimisation; EGO; experimental evaluation.

Reference to this paper should be made as follows: Kanazaki, M., Matsuno, T., Maeda, K. and Kawazoe, H. (2016) 'Optimum driving condition for lift creating cylinder by plasma actuators based on wind tunnel evaluation-based design', Int. J. Automation and Logistics, Vol. 2, Nos. 1/2, pp.45-59. 
Biographical notes: Masahiro Kanazaki is an Associate Professor at Graduate School of System Design, Tokyo Metropolitan University. He received his PhD in the Institute of Fluid Science at the Tohoku University, Japan. His research area is in design engineering and aerospace engineering.

Takashi Matsuno is a Lecturer in Graduate School of Engineering at the Tottori University. He received his $\mathrm{PhD}$ in Graduate School of Engineering from the Nagoya University. His research interest includes the wind tunnel test, the plasma actuator and aerospace engineering.

Kengo Maeda is an MS student in Graduate School of Engineering, Tottori University. His research area is in the plasma actuator. He works under the supervision of Dr. Takashi Matsuno and Dr. Hiromitsu Kawazoe.

Hiromitsu Kawazoe is a Professor in the Graduate School of Engineering, Tottori University. He received his $\mathrm{PhD}$ degree in the Graduate School of Engineering from the Nagoya University. His research interest includes the wind tunnel test and aerospace engineering.

This paper is a revised and expanded version of a paper entitled 'Wind tunnel evaluation based design of lift creating cylinder using plasma actuators' presented at the 18th Asia Pacific Symposium on Intelligent and Evolutionary Systems (IES), Singapore, 10-12 November 2014.

\section{Introduction}

Plasma actuators (PAs, shown in Figure 1) are flow control devices that utilise atmospheric pressure discharge (Matsuno et al., 2012, 2008, 2009, 2010); they have gained attention in recent years, because their advantages of being fully electronically driven with no moving parts and having a simple structure and a fast response are potentially ideal for application to subsonic flow control. In Matsuno et al. (2008), the drag around an airfoil can be reduced with installing PAs on the airfoil. In Thomas et al. (2008), the aerodynamic noise from the cylinder model could be reduced by PAs. Thus, PAs have remarkable benefits for the future aircraft design. Such active flow control devices have also potential to control of the circulation around arbitrary objects and produce the lift-creating object even if it is not airfoil geometry.

In this study, the design problem is defined as the optimisation of lift creation via flow circulation controlled by the PAs. A circular cylinder model is used as a model and two PAs are installed. A genetic algorithm (GA)-based efficient design technique was employed with wind tunnel testing to efficiently find the optimum designs. Through the design case, the applicability of the present wind tunnel testing to the multi-parameter design problem was also investigated.

Design problems are often solved by GAs based on numerical simulation, such as computational fluid dynamics (CFD) (Kanazaki et al., 2004; Sato et al., 2013). However, there are several difficulties with solving the flow field around PAs. First, the accuracy of existing simulation methods is still insufficient. Second, the computational cost is very high for design techniques such as GAs. Several days are needed to acquire the results for each case, whereas the actual flow physics finishes in a few seconds. 
To reduce the experimental cost, kriging surrogate model was applied to represent the input/output relationship in the experimental data. This optimisation technique, which is called efficient global optimisation (EGO) (Jones et al., 1998; Jeong et al., 2005), enables the optimisation of global parameters in a small number of experiments while simultaneously obtaining information on the design space. The EGO-based on Kriging surrogate model can find efficiently near global optimum. The EGO-based optimisation is applied to the design problem which employs time-consuming simulation (Kanazaki et al., 2013a, 2013b). The EGO is also used to find the optimum installation condition of the nacelle chine which installed on the engine nacelle to improve low speed performance of an aircraft in the large scale wind-tunnel (Kanazaki et al., 2008). In Kanazaki et al. (2008), design tables were manually updated because model geometries and configurations were also changed manually.

In authors' first attempt (Kanazaki et al., 2014) for PA optimisation, Kriging surrogate model-based GA performs optimisation during a wind tunnel experiment in real time. The design system is automated developing the interface between the optimisation and the wind tunnel testing. Using developed the wind tunnel evaluation-based optimisation system, the drag reduction around the semi-circular cylinder has been conducted with two parameters. In the authors' study (Kanazaki et al., 2015), the design methodology is applied to more complex design problem, that is that the lift creation via flow circulation controlled by the PAs. In Kanazaki et al. (2015), the optimum design space has been investigated. In this study, the lift creation circular cylinder by PAs is considered to acquire the PA conditions which provide higher lift based on modified design space defined by the design information by previous study. The applicability of the developed system to the multi-parameter design problem was also investigated.

Figure 1 Schematic of plasma actuator

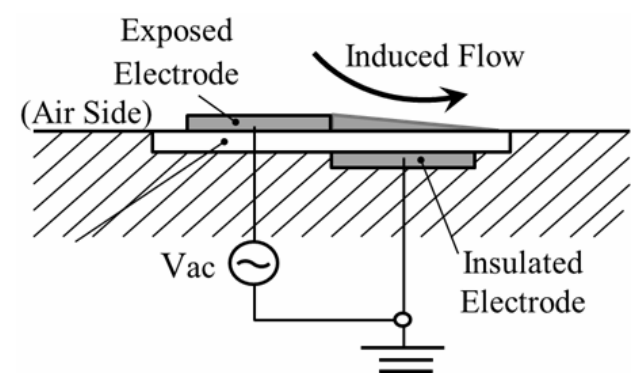

\section{Overview of active flow control by means of plasma actuator}

In this research, PAs consisting of an exposed electrode and insulated electrode (Matsuno et al., 201) were used. A non-conductor was placed between the two electrodes, andAC voltage was applied. Figure 1 shows the setup; this type of PA is called a single dielectric barrier discharge (SDBA) PA. The flow around the PA can be controlled by changing the number and location of PAs and the waveform of the AC voltage. Thus, the optimal technique for solving the design problem has to handle many parameters to acquire the best flow control. 
Generic home-style AC voltage has a waveform with a constant frequency. However, several studies have reported that pulse width modulation (PWM) is effective for flow control of PAs. PWM is a drive system that turns the AC voltage on or off, as shown in Figure 2. The frequency of on/off is defined as the 'modulation frequency' and is expressed by following equation:

$$
f_{\text {mod }}=\frac{1}{T_{1}}[\mathrm{~Hz}]
$$

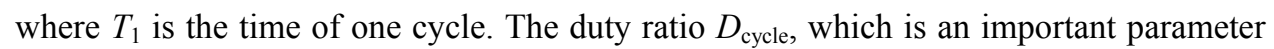
for PWM, is expressed by the following equation:

$$
D_{\text {cycle }}=100 \frac{T_{2}}{T_{1}}[\%]
$$

where $T_{2}$ is the time the AC voltage is on. In this study, $f_{\text {mod }}$ was generated from the base frequency $f_{p}$ as follows.

$$
f_{\text {mod }}=\frac{f_{\mathrm{p}}}{20 x_{\mathrm{m}}}[\mathrm{Hz}]
$$

Equation (3) shows that $T_{1}$ is defined by multiples of the time of base frequency $1 / f_{\mathrm{p}}$. Namely, $f_{\text {mod }}$ is determined by $x_{\mathrm{m}}$. In this study, $x_{\mathrm{m}}$ and $D_{\text {cycle }}$ were considered to be design variables.

Figure 2 Power supply by means of PWM

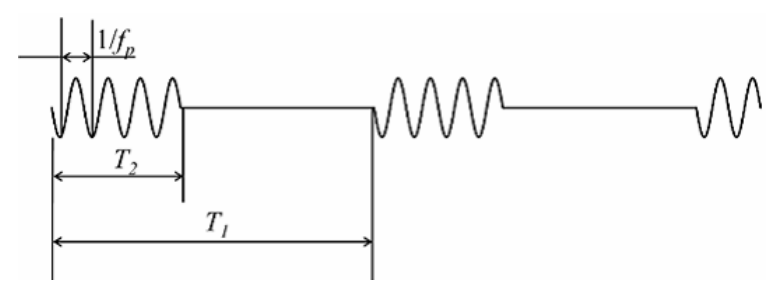

\section{Design method: EGO}

\subsection{Efficient global optimisation}

The optimisation procedure (Figure 3) for PA design based on the wind tunnel evaluation consists of the following steps. First, $N$ design samples are selected by Latin hypercube sampling (LHS) (Jones et al., 1998; Jeong et al., 2005; Kanazaki and Jeong, 2007; Kanazaki et al., 2008), which is a space filling method, and then assessed for the construction of an initial Kriging surrogate model. Second, an additional design sample is added, and the design accuracy is improved by constructing a Kriging model based on all $N+1$ samples. Note that the additional sample is selected by using expected improvement (EI) maximisation (Jones et al., 1998; Jeong et al., 2005; Kanazaki and Jeong, 2007; Kanazaki et al., 2008). GA is applied to solve this maximisation problem. This process is iterated until the improvement of the objective functions becomes 
negligible. Through the design procedure proposed in this paper, all samples are evaluated by the wind tunnel testing. Each technique of the optimisation procedure is described in detail in the following sections.

Figure 3 Optimisation procedure based on wind tunnel evaluation

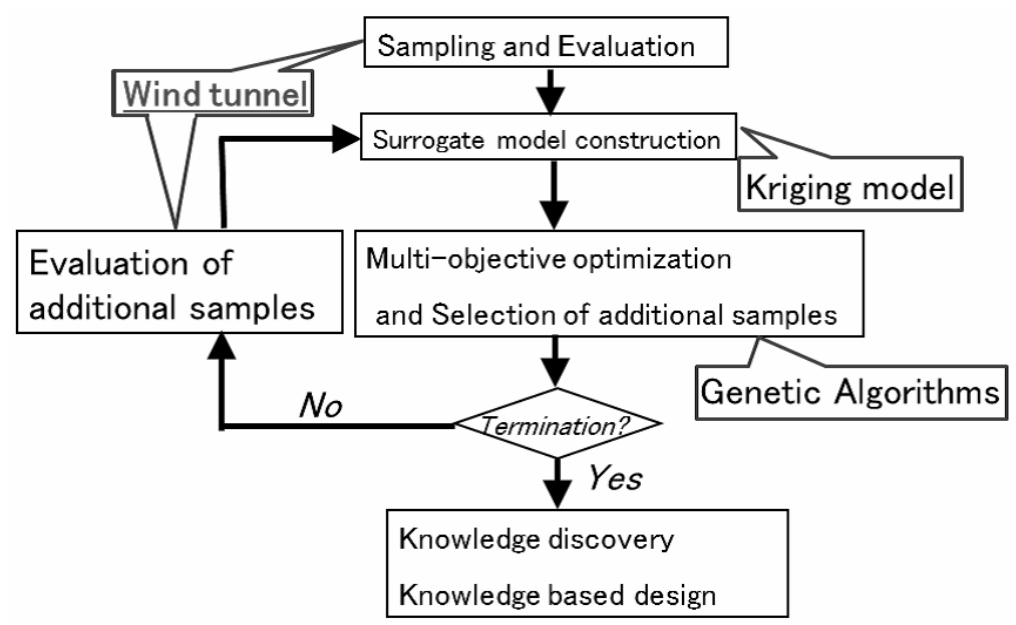

Figure 4 Improvement of the global model by EI maximisation

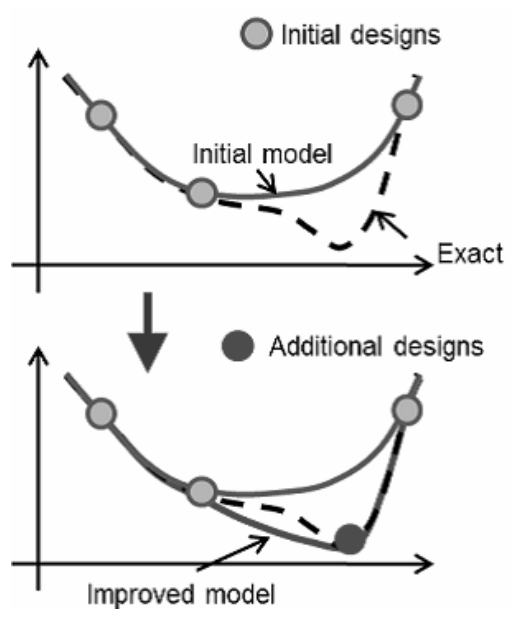

\subsubsection{Kriging model}

The kriging models express the value $y\left(x_{i}\right)$ at the unknown design point $x_{i}$ as

$$
y\left(x_{i}\right)=\mu+\epsilon\left(x_{i}\right) \quad(i=1,2, \ldots, m)
$$

where $m$ is the number of design variables, $\mu$ is a constant of the global model, and $\epsilon\left(x_{i}\right)$ represents a local deviation from the global model. The correlation between $\epsilon\left(x_{i}\right)$ and $\epsilon\left(x_{j}\right)$ is strongly related to the distance between the corresponding points, $x_{i}$ and $x_{j}$. In the 
Kriging models, the local deviation at an unknown point $\mathrm{x}$ is expressed using stochastic processes. Specifically, a number of design points are calculated as sample points and then interpolated using a Gaussian random function as the correlation function to estimate the trend of the stochastic process.

\subsubsection{Expected improvement}

Once the models are constructed, the optimum point can be explored using an arbitrary optimiser. However, it is possible to miss the global optimum design, because the approximate model includes uncertainty. Therefore, this study introduced EI values as the criterion. This study solves the lift maximisation problem, then EI for maximisation problem can be calculated as follows.

$$
E[I(x)]=\left(f_{\max }-\hat{y}\right) \Phi\left(\frac{f_{\max }-\hat{y}}{s}\right)+s \phi\left(\frac{f_{\max }-\hat{y}}{s}\right)
$$

where $f_{\max }$ is the minimum values among sample points, $s$ is root mean square error (RMSE) and $\hat{y}$ is the value predicted by equation (4) at an unknown point $x$. $\Phi$ and $\phi$ are the standard distribution and normal density, respectively. EI considers the predicted function value and its uncertainty, simultaneously. Therefore, by selecting the point where EI takes the maximum value, as the additional sample point, robust exploration of the global optimum and improvement of the model can be achieved simultaneously as shown in Figure 5 because this point has a somewhat large probability to become the global optimum. In this study, the maximisation of EI is carried out using GA expressed as following section.

\subsubsection{Genetic algorithm}

GAs [Figure 5(a)] was first proposed by Holland in the early 1970s (Holland, 1975) and are based on the evolution of living organisms with regard to adaptation to the environment and the passing on of genetic information to the next generation. GAs can find a global optimum because they do not use function gradients, which often lead to an exact local optimum. Thus, GA is a robust and effective method that can handle highly nonlinear optimisation problems involving non-differentiable objective functions. Owing to this advantage, GAs was applied to this experimental system. The GA used in this study (Kanazaki et al., 2004) utilises a real-coded representation, the blended crossover $(\mathrm{BLX}-\alpha)$, and the uniform mutation. The selection probability of individuals for the crossover and mutation is expressed as follows.

$$
\text { prob }=c(1-c) \text { rank } 1-1.0
$$

where rank is the value of fitness ranking among the population.

In BLX- $\alpha$, children are generated in a range defined by the two parents as shown in Figure $5(\mathrm{~b})$. The range is often extended equally on both sides as determined by the parameter $\alpha$.

The developed system uses GA with an island model [Figure 5(c)], which is a distributed population scheme (Kanazaki et al., 2004; Hiroyasu et al., 2000); it has a mechanism for global searching to avoid convergence at local optima. This model divides the population into sub-populations called islands. To retain a high degree of diversity 
and avoid early convergence, some individuals in each sub-population are moved to other sub-populations (migration) every $k$ generations.

Figure 5 Schematic illustration of GA with the distributed scheme, (a) flowchart of GA (b) BLX- $\alpha$ (c) island model

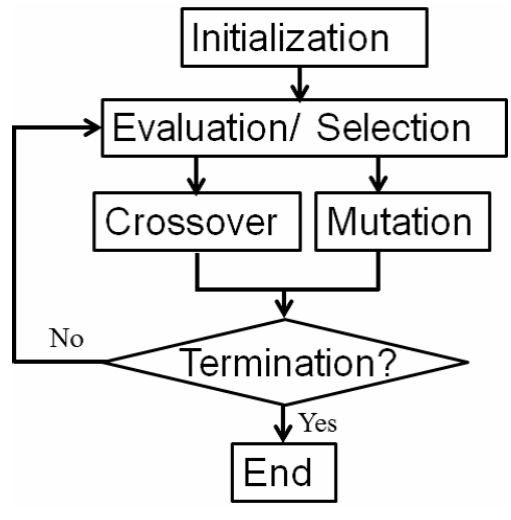

(a)

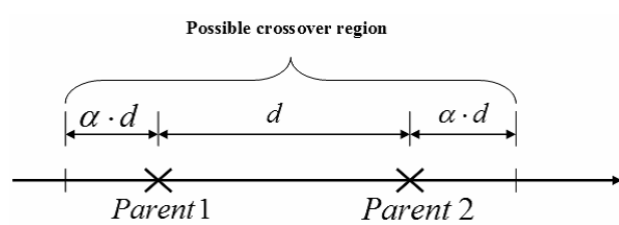

(b)

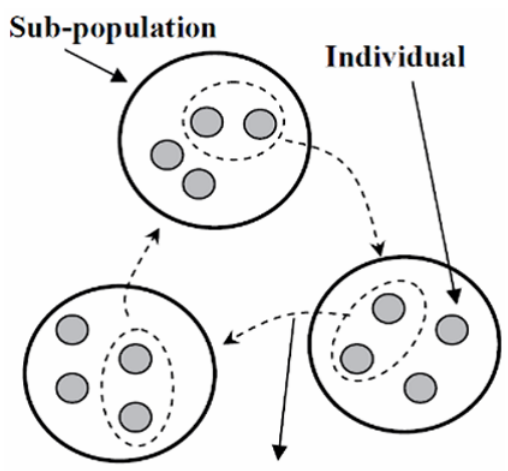

Migration

(c)

\subsection{Knowledge discovery techniques}

\subsubsection{Functional analysis of variance}

A functional analysis of variance (functional ANOVA) which is one of the multivariate analyses is carried out to differentiate the contributions to the variance of the response from the model. To evaluate the effect of each design variable, the total variance of the model is decomposed into that of each design variable and their interactions. The decomposition is accomplished by integrating variables out of the model $\hat{y}$. The main effect of design variable $x_{i}$ is as follows.

$$
\mu_{i}\left(x_{i}\right) \equiv \int \cdots \int \hat{y}\left(x_{1}, \cdots, x_{n}\right) d x_{1}, \cdots d x_{i-1}, d x_{i+1}, \cdots, d x_{n}-\mu
$$

where the total mean $\mu$ is calculated as 


$$
\mu \equiv \int \cdots \int \hat{y}\left(x_{1}, \cdots, x_{n}\right) d x_{1}, \cdots d x_{n}
$$

The proportion of the variance attributed to the design variable $x_{i}$ to the total variance of the model can be expressed as:

$$
p \equiv \frac{\int\left[\mu_{i}\left(x_{i}\right)\right]^{2} d x}{\int \cdots \int\left[\hat{y}\left(x_{1} \cdots x_{n}\right)-\mu\right]^{2} d x_{1} \cdots d x_{n}}
$$

The value obtained by equation (9) indicates the sensitivity of an objective function to the variance of a design variable.

\subsection{Scatter plot matrix}

The solution and the design space of the multivariable design problem obtained by EGO are observed by the scatter plot matrix (SPM) which is one of the data mining, because the Kriging model cannot be visualised directly when the design problem has over four attribute values. SPM arranges two-dimensional scatter plots like a matrix among the objective functions and the design variables and facilitates the investigation of the design problem investigation. Each of the rows and columns is assigned attribute values such as design variables, objective functions, and constraint values. The diagonal elements show mutual same plots. Therefore, it can be said that the SPM shows scatter plots on the upper triangular part of the matrix and the correlation coefficients on the lower triangular part as additional information. Interactive Scatter Plot Matrix (iSPM) ver. 2.0 (Oyama, 2011) developed in Japan Aerospace Exploration Agency is employed in this study.

\section{Experimental setup}

\subsection{Wind tunnel and model}

The wind tunnel experiments were conducted in the subsonic closed-return wind tunnel of the Aerodynamics Laboratory at Tottori University. The wind tunnel has a closed test section with a $0.70 \mathrm{~m} \times 1.0 \mathrm{~m}$ cross-section and $2.0 \mathrm{~m}$ length (Figure 6). A two-dimensional circular model $(105 \mathrm{~mm}$ in diameter) was used as shown in Figure 7. Model was placed on a flat plate and mounted to a support connected to a six-component external balance for measurement of the aerodynamic forces and moments. The output of the balance was amplified and acquired with a data acquisition board (National Instruments PXI-8106). The output signal contains noise from the atmospheric discharge of the plasma actuators. To eliminate this effect, the clean portion of the signal, during which the discharge did not appear, was extracted and used as a 'clean' portion of the data. 
Figure 6 Test section of the wind tunnel

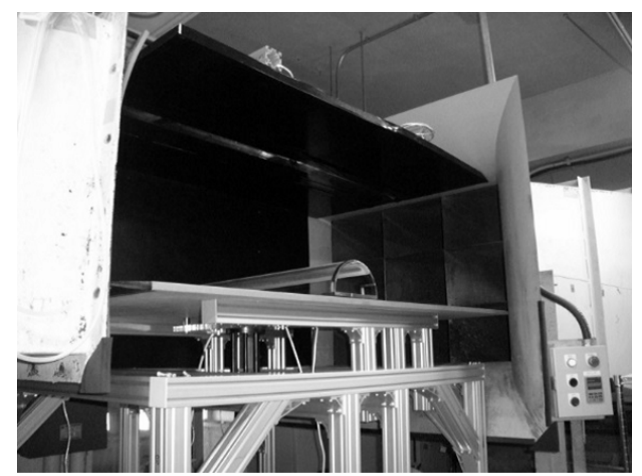

\subsection{PA and its power supply}

In this study, two PAs were installed on the surface of the model. PA\#1 and PA\#2 were installed with mount angles of $\theta 1=85.0^{\circ}$ and $\theta 2=-85.0^{\circ}$, respectively, as shown in Figure 7. The reference waveform of a high-voltage AC input was amplified by a solid-state high-power amplifier; the input power was increased up to $400.0 \mathrm{~W}$ with amplitude of 70.0 Vpp. A high-voltage transformer was used to achieve an AC input with amplitude of up to $30 \mathrm{kV}$ at a frequency of $5.0-15.0 \mathrm{kHz}$. The voltage and current of the $\mathrm{AC}$ input were monitored by an oscilloscope along with the reference waveform.

Figure 7 Circular cylinder model and the location of plasma actuators (see online version for colours)
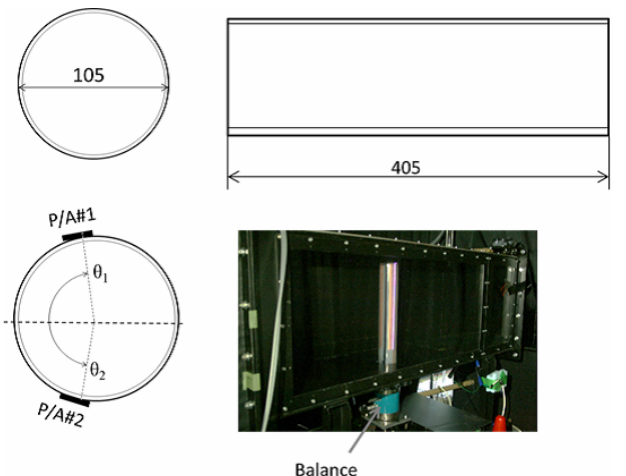

\subsection{Integration of experiment system}

Figure 8 shows the schematic illustration of the developed system. EGO is executed in the workstation and receives the experimental data via LabVIEW® from the balance in the wind tunnel. The condition of the AC voltage can be automatically set during the optimisation process based on balance measurements. 
Figure 8 Schematic diagram of the integrated optimisation system

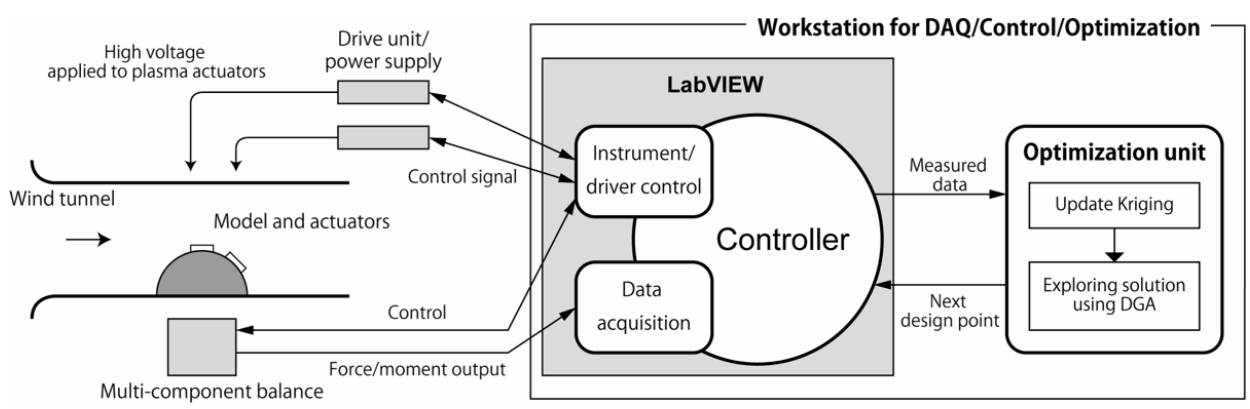

Figure 9 Software control panel

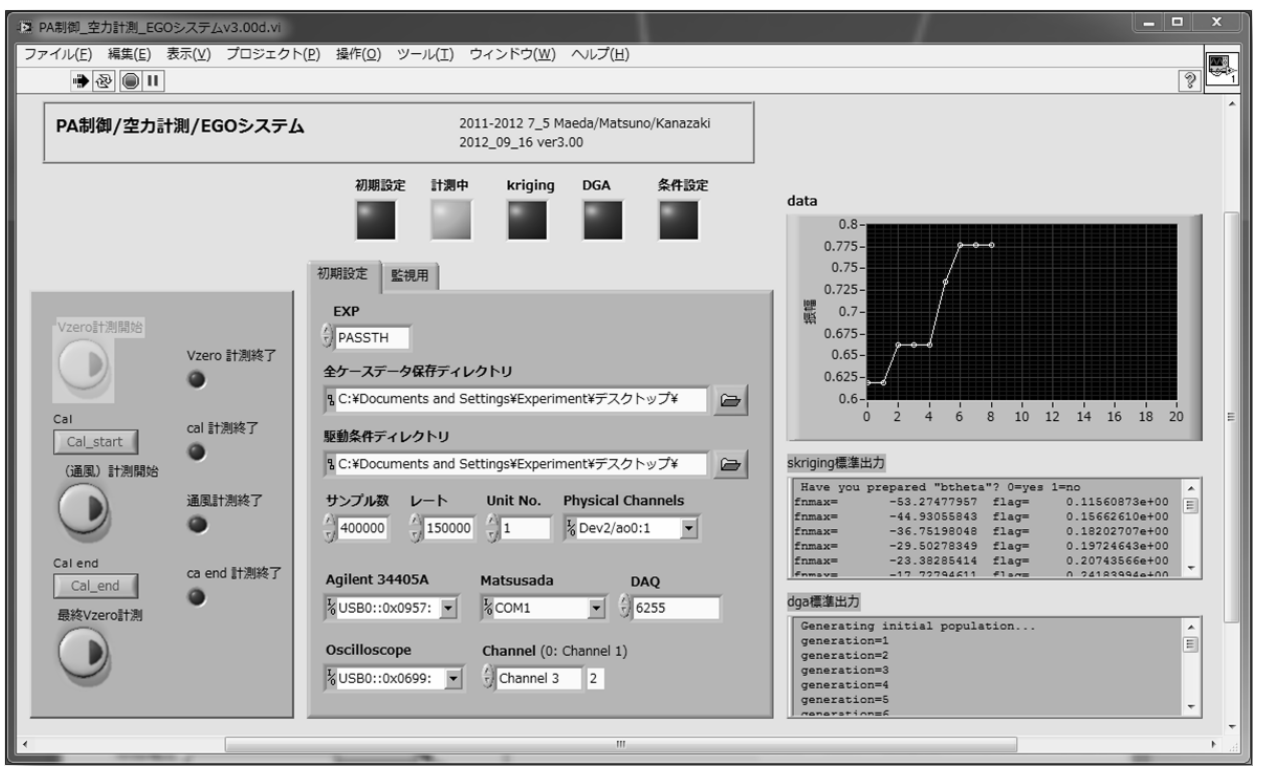

\section{Formulation}

In this study, multi-parameter design problem which has four design variables was considered and the lift creation effect due to circulation control by PAs was investigated. The objective function was maximisation of the lift coefficient $\left(C_{1}\right)$ around the circular cylinder model. This design problem can be expressed as follows.

$$
\text { Maximise } C_{1}
$$

The flow velocity was set to $10.0 \mathrm{~m} / \mathrm{s}$. Equation (5) can be written for the present design problem as follows.

$$
E I_{C l}=\left(C l_{\max }-\hat{y}\right) \Phi\left(\frac{C l_{\max }-\hat{y}}{s}\right)+s \phi\left(\frac{C l_{\max }-\hat{y}}{s}\right)
$$


where $C l_{\max }$ is the maximum values among sample point.

The design problem expressed in equation (10) was solved by changing four parameters $\left(x_{\mathrm{m}}, D_{\text {cycle1 }}, D_{\text {cycle2 }}, \phi\right)$ related to the AC voltage waveform. In this case, two

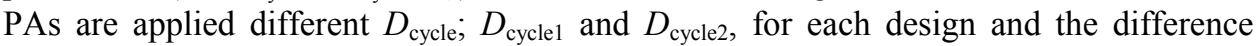
between $D_{\text {cycle1 }}$ and $D_{\text {cycle } 2}$ is decided by a phase difference $\phi$. The design space is defined as follows.

$$
\left\{\begin{array}{l}
30.0 \leq f_{\mathrm{mod}} \leq 200.0[\mathrm{~Hz}] \\
0.0 \leq D_{\text {cycle } 1} \leq 50.0[\%] \\
0.0 \leq D_{\text {cycle } 2} \leq 50.0[\%] \\
-90.0 \leq \phi \leq 90.0[\mathrm{deg} .]
\end{array}\right.
$$

$\phi$ is the phase difference between PA\#1 and PA\#2. Consequently, the time lag can be expressed as $\phi=f_{\text {mod }}$.

\section{Results}

\subsection{Design exploration result}

In this section, the design problem expressed by equation (10) is discussed. To construct the initial Kriging model, 15 samples were obtained by LHS. To acquire additional samples, the island GA was executed with the following specifications: BLX-0.5 $(\alpha=0: 5)$, four subpopulations, 16 individuals for each subpopulations (64 individuals generated in total) and 64 generations. The EGO process will be stopped after five or more additional samples show better function value than that of initial samples (Kanazaki et al., 2008, 2013a, 2013b).

After the objective function was converted, 16 additional samples were obtained, for a total of 31 sample designs. Figure 10 shows the history of $C_{1}$ values for the sampling process. According to the history, the objective function converged well.

Figure 10 Progression of objective function with sample number for the lift maximisation problem (see online version for colours)

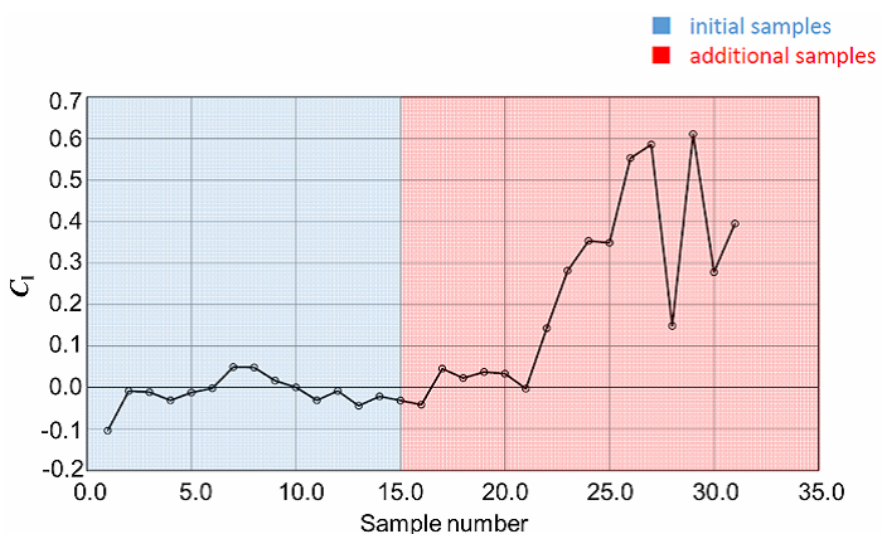




\subsection{Design knowledge by analysis of variance}

Figure 11(a) shows the main effects and the two-way interaction of the design variables for objective function in this design problem. According to Figure 11(a), $D_{\text {cycle2 } 2 \text {, which }}$ defines the duty ratio for PA on the lower side of the cylinder, has a predominant influence on $C_{1}$. In addition, main effect of $D_{\text {cycles }}$ and two-way interaction of $D_{\text {cycle } 1}-D_{\text {cycle } 2}$ are effective to $C_{1}$. These results suggest that the circulation which creates aerodynamic lift around the model is decided by duty ratio. On the other hand, $x_{\mathrm{m}}$ and $\phi$ do not have influence in this analysis.

Figure 11 Functional ANOVA results, (a) the main effects and the two-way interaction effect of design variables of the design variables for objective function (b) variance of $C_{1}$ by design variables (see online version for colours)

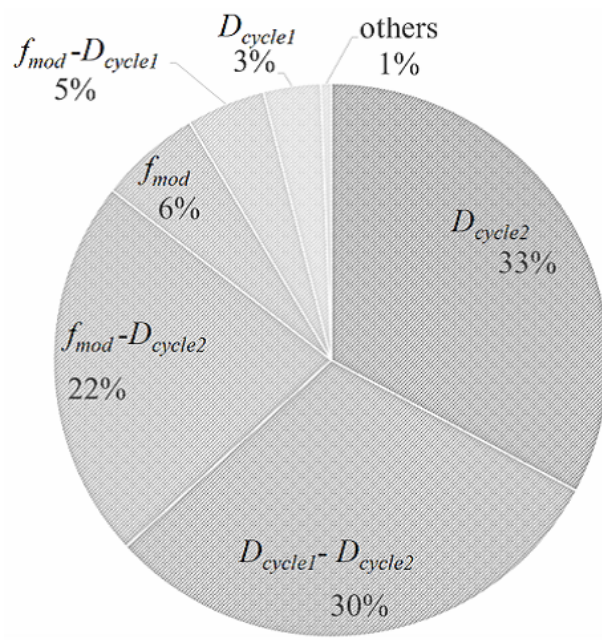

(a)

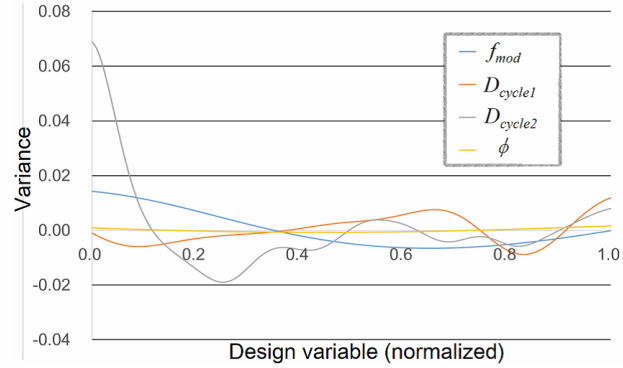

(b)

Figure 11(b) shows variances by design variables calculated by equation (7). (The horizontal axis is normalised design variables by minimum/maximum variables in design space, $d v s_{\text {norm. }}$.) According to Figure $11(\mathrm{~b})$, it is found that $C_{1}$ shows higher when normalised $D_{\text {cyclel }}$ is approximately $50 \%$ and $100 \%$. It suggests that such duty ratio can accelerate the flow on the upper side of the model and create suction. High duty ratio requires high electronic energy, because the energising time is higher. Thus, if the lower driving cost is required, the designer should select $50 \%$ normalised $D_{\text {cyclel }}$. $C_{1}$ also shows the highest value when normalised $D_{\text {cycle } 2}$ is approximately $0 \%$. The flow on the lower side should not be accelerated by the PAs volume force, because high pressure on the lower side is required to create the lift. While $0 \%$ normalised $D_{\text {cycle2 }}$ achieves higher $C_{1}$, it is also remarkable that the $C_{1}$ is multi-modal along normalised $D_{\text {cycle2 }}$.

\subsection{Visualisation of design problem by SPM}

Figure 12 shows the visualisation results obtained by SPM. According to Figure 12(a), which shows the scatter plot for all parameter combinations, $D_{\text {cycle2 }}$ of the additional samples which achieve higher $C_{1}$ always became lower. This result agrees with 
Figure 12(b). On the other hand, there were no unique values of $x_{\mathrm{m}}$ and $D_{\text {cycle }}$ that maximised $C_{1}$. It suggests that the PAs can work well for the upper side (suction side) compared with the lower side (pressure side).

Figure 12(b) is the response surface for $C_{l}$ regarding $D_{\text {cycle1 }}$ and $D_{\text {cycle2, which are }}$ effective design variables from Figure 11(a). Figure 11 corresponds to a matrix element which is the second row and the third column of Figure 12. The global trend can be visualised using the model shown in Figure 12(b); that is, a lower $D_{\text {cycle2 }}$ can potentially yield a higher $C_{1}$. In addition, $D_{\text {cycle }}$ should be approximately $22 \%$ to increase $C_{1}$. With a such higher $D_{\text {cycle } 1}$, a higher total energy for the AC voltage is required for PA\#1. This is reasonable as a higher $D_{\text {cycle }}$ induces greater acceleration of the flow on the upper side of the model, that is, the suction side. Furthermore, $C_{1}$ can be increased with this range while the total electrical energy is relatively low because lower $D_{\text {cycle1 }}$ (approximately $40 \%$ ) also achieves high $C_{1}$. This suggests that the PAs can control the flow for lift creation with minimal electrical energy and proper PWM driving conditions.

Figure 12 Visualisation of the lift maximisation problem using SPM, (a) scatter plot of all parameters (b) response surface regarding $D_{\text {cyclel }}$ and $D_{\text {cycle2 }}$, coloured according to $C_{l}$ (b) the horizontal axis is normalised design variables by minimum/maximum variables in design space, $d v s_{\text {norm }}$ (see online version for colours)

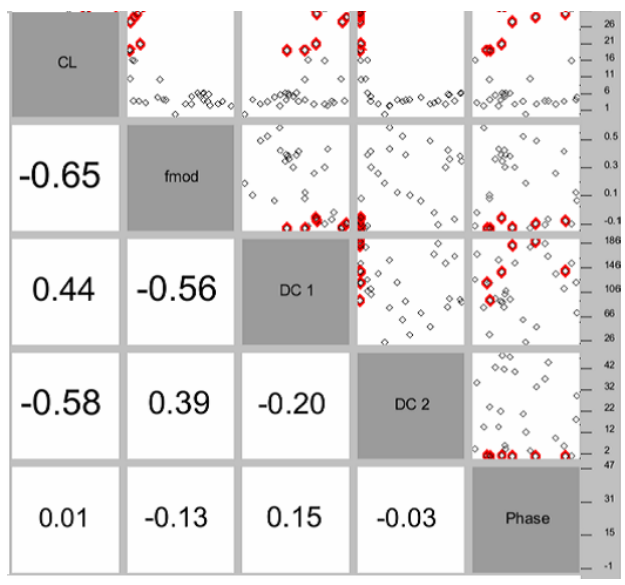

(a)

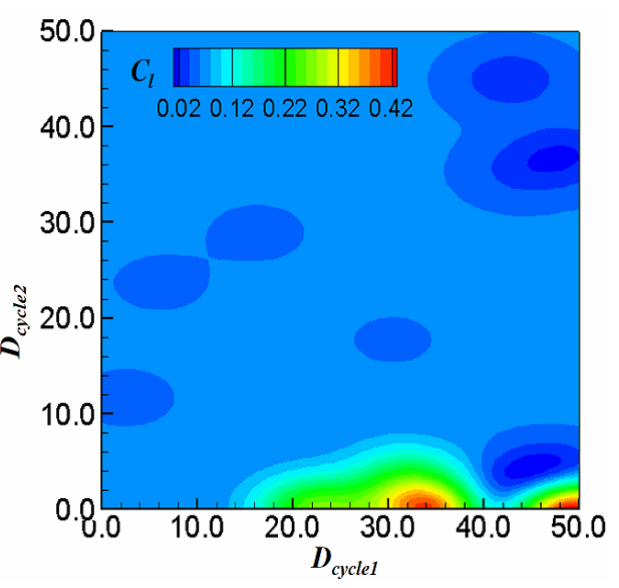

(b)

\section{Conclusions}

Aerodynamic control performance of plasma actuators was optimised using wind tunnel test-based EGO. In this study, the lift-creating cylinder using plasma actuators is considered. This problem was that the circulation around a circular cylinder model was controlled to maximise the lift around the model. The optimisation technique is firstly integrated in the operating system of the wind tunnel experiment to enable automation of the data acquisition/optimisation process. Using the developed system, lift maximisation problems were solved. After several additional samples are obtained, the analysis of variance and the parallel coordinate plot is employed for the knowledge 
discovery. Using these techniques, it is found that duty ratios for two plasma actuators have the dominant effect for this problem. It is also found that the lower duty ratio is required for the plasma actuator installed on the lower side of the cylinder and the response of the lift coefficient along the duty ratio of the plasma actuator installed on the upper side is the multi-modal.

\section{References}

Hiroyasu, T., Miki, M. and Watanabe, S. (2000) 'The new model of parallel genetic algorithm in multiobjective optimization problems (divided range multi-objective genetic algorithm)', IEEE Proc. the Congress on Evolutionary Computation, Vol. 1, pp.333-340.

Holland, J.H. (1975) Adaptation in Natural and Artificial Systems, University of Michigan Press, Ann Arbor.

Jeong, S., Murayama, M. and Yamamoto, K. (2005) 'Efficient optimization design method using kriging model', Journal of Aircraft, Vol. 42, No. 2 pp.413-420.

Jones, D.R., Schonlau, M. and Welch, W.J. (1998) 'Efficient global optimization of expensive black-box function', Journal of Global Optimization, Vol. 13, pp.455-492.

Kanazaki, M. and Jeong, S. (2007) 'High-lift airfoil design using kriging based moga and data mining', Korea Society for Aeronautical and Space Sciences International Journal, Vol. 8, No. 2, pp.28-36.

Kanazaki, M., Hanida, R., Nara, T., Shibata, M., Nomura, T., Murayama, M. and Yamamoto, K. (2013a) 'Challenge of design exploration for small blended wing body using unstructured flow solver', Computers \& Fluids, October, Vol. 85, pp.71-77.

Kanazaki, M., Takagi, H. and Makino, Y. (2013b) 'Mixed-fidelity efficient global optimization applied to design of supersonic wing', Procedia Engineering, Vol. 67, No. 1, pp.85-99.

Kanazaki, M., Matsuno, T., Maeda, K. and Kawazoe, H. (2014) 'Efficient global optimization applied to wind tunnel evaluation based optimization for improvement of flow control by plasma actuator', Engineering Optimization, Vol. 46, No. 12 pp.1-17.

Kanazaki, M., Matsuno, T., Maeda, K. and Kawazoe, H. (2015) 'Wind tunnel evaluation based design of lift creating cylinder using plasma actuators', Proceedings of the 18th Asia Pacific Symposium on Intelligent and Evolutionary Systems, Vol. 1, pp.663-677.

Kanazaki, M., Obayashi, S. and Nakahashi, K. (2004) 'Exhaust manifold design with tapered pipes using divided range MOGA', Engineering Optimization, Vol. 36, No. 2, pp.149-164, Taylor \& Francis.

Kanazaki, M., Yokokawa, Y., Murayama, M., Ito, T., Jeong, S. and Yamamoto, K. (2008) 'Nacelle chine installation based on wind tunnel test using efficient design exploration', Transaction of Japan Society and Space Science, November, Vol. 51, No. 173, pp.146-150.

Matsuno, T., Kawaguchi, M., Fujita, N., Yamada, G. and Kawazoe, H. (2012) 'Jet vectoring and enhancement of flow control performance of trielectrode plasma actuator utilizing sliding discharge', 6th AIAA Flow Control Conference, AIAA-Paper 2012-3238.

Matsuno, T., Kawazoe, H. and Corke, T.C. (2008) 'Forebody vortex control on high performance aircraft using PWM-controlled plasma actuators', 26th Congress of International Council of the Aeronautical Sciences, ICAS 2008-3.3.4.

Matsuno, T., Kawazoe, H. and Nelson, R.C. (2009) 'Aerodynamic control of high performance aircraft using pulsed plasma actuators', 47th AIAA Aerospace Sciences Meeting Including The New Horizons Forum and Aerospace Exposition, AIAA paper 2009-0697. 
Matsuno, T., Ota, K., Kanatani, K. and Kawazoe, H. (2010) 'Parameter design optimization of plasma actuator configuration for separation control', 5th Flow Control Conference, AIAA paper $2010-4983$.

Oyama, A. (2011) 'Design innovation with multiobjective design exploration' [online] http://flab.eng.isas.jaxa.jp/monozukuri/mode/english/index.html (accessed 16 August).

Sato, T., Kanazaki, M., Yotsuya, Y. and Matsushima, K. (2013) 'Parametric airfoil representation toward efficient design knowledge discovery in various flow condition', Proc. the AsiaPacific International Symposium on Aerospace Technology (APISAT), CD-ROM, Takamatsu, Japan.

Thomas, F.O., Kozlov, A. and Corke, T.C. (2008) 'Plasma actuators for cylinder flow control and noise reduction', AIAA Journal, Vol. 46, No. 8, pp.1921-1931. 